\title{
Research of Mine-shaft Wireless Sensor Network Based on Agent
}

\author{
Wu Li Song ${ }^{1, a^{*}}$ and Jing Qiao ${ }^{1}$ \\ ${ }^{1}$ College of Information Engineering,Taishan Medical University,Taian,Shandong 271016,China \\ a *Authors to whom correspongence should be addressed.Electronic addresses:songswl@163.com
}

Keywords: Wireless sensor networks; Agent; Distributed sensor network; Network communication; Mine-shaft safety.

\begin{abstract}
In order to ensure the safety in production,it is necessary to build a wireless communication system that does not rely on a wire backbone.With the development of wireless sensor network, this kind of monitoring system has been true.With the emergence and development of agent,the idea distributed sensor network based on agent has been raised,and the methods of performing collaborative task between many sensor nodes have been developed.It has reduced the requirement of bandwidth and the energy expenditure of network,thereby increasing the life of WSN.

With the rapid development of sensor technology, embedded computing technology, communication technology and semiconductor and microelectronics system manufacturing technology, micro sensor with sensing, computing and communication capabilities of the store began to appear in the military, industrial, agricultural and aerospace fields[1]. Wireless sensor networks is Set the sensor actuator, a controller and a communication device into integration, resource constrained embedded devices in sensing and driving control ability, computing ability, communication ability in a body. Wireless sensor network is composed of these micro sensor can be real-time monitoring, sensing and collecting network of regional distribution.A variety of monitoring object information, and the information is processed, transmitted to the users who need the information. In recent years, a large number of coal mine production safety in academic and high-tech results continue to emerge, greatly improve the safety production level of coal mine. Coal mine safety monitoring system in cable. Play a decisive role in safety production of coal mine, however, because the cable monitoring system its own limitation and the complexity of coal mine, some environmental parameters under mine, such as methane, dust, carbon monoxide, negative pressure, temperature, wind speed can effectively obtain monitoring, causing great security risks[2]. If we combine the wireless monitoring system based on wired monitoring of existing systems, which will effectively make up for the lack of existing monitoring system, improve the coal mine safety production level of great.
\end{abstract}

\section{The development of wireless sensor network}

Sensor network technology from generation to now, can be summarized as four stages of development [3].20 century 70 time, appear using traditional sensor network model of the point of the cable data transmission point and sensor controller, called the first generation of sensor networks. After show the second generation interface connection, serial port (such as RS232, RS485), data transmission network model of parallel connection. To meet the interconnection and integration of a large number of sensor monitoring system, and then developed a sensor network structure based on field bus technology, increase the capability of processing information 
integrated,Known as the third generation of sensor network. From the beginning of the 90's of last century, people began to build intelligent wireless network, will be collected by each sensor information through wireless transmission, resulting in a new type of sensor network -- wireless sensor network, which is called the fourth generation sensor networks Wireless sensor network (WSN) development, from the beginning in can be divided into three stages. The first stage [4], mainly is the research and development of hardware, the development and the development of various wireless sensor nodes are committed to the miniaturization, low cost, low power consumption, emerged in a number of new wireless sensor Nodes, such as American in 1999 the development of "smart dust" (Smart Dust) project. The second stage, the focus is on the study of the specific implementation of wireless communication networks, including network architecture, communication protocol and so on. The third stage, is mainly to the large number of nodes in wireless sensor network intelligent cluster system, Research and practical application. At present, the research on wireless sensor network is in the second and third stages, it will bring far-reaching influence to human life and production. The development of wireless sensor network is very popular in the developed countries, American military earliest start without wireless sensor network technology ,Research of project operation in wireless sensor networks as one of the key development industry[4]. The wireless sensor network as the current information technology an important frontier technology, its large-scale application is an inevitable trend, it will bring great changes to human society. But at present the application situation, the development of wireless sensor network is also proposed to seek higher, as more of the low cost, the more standard technology, technical agreement and mature. From the current situation of wireless sensor network overall standardization degree low, common IEEE802. 15.4,ZigBee, IEEE1451 and IPv6 family of standards and a network of interconnected Draft, technical standards will be an important task wireless sensor networks in the future development[5].

\section{The characteristics of Wireless Sensor Networks}

The main feature of wireless sensor networks is the use of a large number of wireless nodes working together to complete the monitoring of the monitoring area monitoring objects. These wireless nodes usually is composed of wireless sensor nodes (Sensor node), the sink node (Sink node) and the management node (Manage Node).

Compared with the traditional wireless network has the following characteristics:

The limited resource nodes usually have limited processing speed, limited storage space, limited communication bandwidth and node energy is limited, once the battery energy depletion, nodes will stop working.

A number of nodes. A large number of nodes are put into the same region execution monitoring tasks, the use of high connectivity between nodes to ensure network system resistance to destroy and fault tolerance.

Nodes are prone to failure. Because the energy is limited, in extreme circumstances, individual nodes are prone to failure.

Data centric nodes to total bureau. Not only the IP address to identify, only need to label identification using local can be distinguished.

The self-organizing and adaptive ability. Due to the limitation and energy saving energy needs, nodes need to automatically join and leave the network, and switch between work and sleep. Therefore, wireless sensor network should have the ability of self-organization and adaptation.

Multi hop routing. Completed by ordinary nodes, the communication distance of nodes in the network is generally in the range of tens to hundreds of meters, the need for data communication 
nodes through intermediate network nodes and the radio range outside of the node, and long distance communication energy consumption is relatively large. Therefore, considering the transmission reliability and energy saving data need to adopt multi hop communication mode.

The wireless dynamic topology. Sensor network is a dynamic network, nodes can be mobile. Nodes can exit the network at any time, can also be added to the current network.

\section{Research and application in coal mine monitoring equipment in wireless sensor network}

The sensor module of wireless sensor network node can include various types of sensors, such as heat, vibration, sound, image, electromagnetic, infrared, these sensors can monitor various equipment and environmental parameters, including: speed, vibration, pressure, temperature and humidity, light intensity, gas composition, so monitoring in military personnel positioning and gas monitoring system of wireless sensor network.

The main technology of coal mine underground working surface equipment and the research status and the use of environmental monitoring

At present, for the application of wireless sensor network in the underground working surface equipment and environment monitoring and diagnosis system analysis and research is not complete. In the monitoring region, using the self-organizing way constitute a sensor network. Because of the condition of underground working face is very bad, and contain explosive gases, and shearer, hydraulic support node installation size is limited, so the request node volume can not be too big and explosion-proof.

The key technology of the system. Wireless communication technology, wireless communication. Several common modes of infrared communication (IrDA), Bluetooth communication (Bluetooth), Wi Fi , IEEE802. 15.4 IEEE802. 15.4, which is for low rate wireless communication standard of the low energy consumption, low cost, as the main target of design, many research institutions put it as a wireless communication platform based on wireless sensor network. But in terms of wireless physical layer, the electromagnetic wave is not the best choice of physical layer transmission mode of underground wireless sensor networks. One of the physical layer technology of electromagnetic waves outside the magnetic induction Magnetic Induction can also be considered in addition. The magnetic induction transmission with different medium magnetic permeability and multiple path almost as the electromagnetic wave transmission, does not appear to decline phenomenon and can meet the advantages of miniaturized antenna. At present the magnetic induction by FSK modulation may be under water communication hundreds of meters. Magnetic induction technology in the carrier frequency of $100 \mathrm{KHZ}$ has also been demonstrated for use in underground communication.

The embedded server technology. Embedded system is to be embedded into a variety of products or in the engineering of software and hardware of the system to the microprocessor as the core of embedded system. Combining with Internet technology, formed with the embedded web server, data acquisition, data processing, protocol conversion, remote IE access function. Using the embedded server technology can be processing monitoring data of the underlying wireless sensor networks, and protocol conversion, through the underground industrial Ethernet transmission to the ground monitoring center. So the embedded server technology is realized by households and the underground wireless monitoring network communication portals.

Network topology control. Have a great impact on network topology of wireless sensor network energy consumption and cost of the network of networks. Wireless sensor network is a self-organizing network topology control has a special significance to the automatic generation of 
the network topology. Therefore, control and optimization of network topology, is very important to balance the node energy consumption and cost.

Routing protocol. Routing protocol is one of the key technologies of wireless sensor networks, its main role is to data packets from the source node to the destination node. The wireless sensor network is a self-organizing network routing protocol design can draw so wireless ad hoc routing protocol research. In the routing design factors wireless sensor network, network characteristics is the main basis for the design of protocol design, is the former provided[5]. Due to the limited hardware resources sensor nodes, routing protocols run on it can not be too complex. In addition, the network energy saving, reliability, scalability and other requirements, these are designed to routing protocol put forward higher requirements.

Network security. Wireless sensor network is a task oriented network, not only for data collection and transmission, but also to carry out data, acquisition and integration, network control. In order to ensure the security of network against malicious attacks in wireless sensor networks need some basic security mechanism, namely guarantee task execution confidential sex, data integrity and transmission security. At present, the realization of the basic security mechanism of wireless sensor network comprises: point-to-point information confirmation, integrity authentication, data encryption and so on.

The time synchronization technology. A key mechanism of time synchronization is a collaborative work system of wireless sensor network, in addition to guarantee the reliable data transmission, the wireless sensor network data processing, sleep scheduling, time stamp and the positioning function also requires the node clock synchronization. In the present time synchronization protocol for wireless sensor networks have many research, comparison a typical reference broadcast synchronization (RBS) protocol, time Diffusion Protocol (TDSP), asynchronous Diffusion Protocol (ADP), time sync protocol for sensor networks (TSPSN) firefly time synchronization technology and development in recent years.

Data fusion technology. Data acquisition equipment information in the use of wireless sensor networks, some of the very large amount of information data, for example the vibration signal. But the communication energy consumption of wireless nodes than the calculated energy consumption high cost by several orders of magnitude. And the current energy is the inherent constraints in wireless sensor, so with limited computation and storage capabilities of sensor nodes in each node itself, data collection and data forwarding, for data fusion to reduce the amount of data transmission is an effective way of energy saving of the existing data. Data fusion technology can reduce the amount of data to be transmitted packets, reducing the Sink node receives probability duplicate data packets, also can reduce the reliability of single node data collection is not, improve the detection results.

Application layer technology. Wireless sensor network application layer is composed of various kinds of application software systems, main research and application layer technology is between the development of various wireless network application software and multi task scheduling, the wireless network to perform a variety of tasks for deployment. TinyOS embedded system of California University R \& D is specifically oriented to the development of wireless sensor networks, code the quantity is small, modular, can meet the requirements of data acquisition, network communication and other functions . The fault diagnosis task oriented working surface equipment and environment monitoring system in the application layer can build intelligent analysis of the collected data, so that more accurate, timely detection of equipment failure, real-time monitoring of the gas content and overrun the alarm. 
Analysis of soft hardware node. Design of hardware platform of wireless sensor node, low power consumption, need to be consistent with the micro volume, low cost etc., and has the characteristics of easy installation, layout, programmable. There are mainly two kinds of design scheme of hardware platform of wireless sensor nodes: the first method combined chip, processor $(\mathrm{MCU})+$ transceiver $(\mathrm{RF})$, the processor can use Atmel Mega128, PIC16, MSP430, transceiver adopts TI TI company's CC2420 or the Ember company's EM240; second systems integrated on-chip with application of SOC, such as the TI TI company CC2430, Jennic company, Ember company JN5121 EM250 and other.Sink node adopts ARM + Linux architecture design. The Sink node is the sink node, is an important node in wireless sensor network, no Sink nodes have no so called wireless sensor networks. The Sink node is responsible for collecting, processing and transmitting data, and is responsible for the collected data through Internet transmitted to the ground monitoring computer, is a gateway node between a monitoring computer and various sensors. So the importance the Sink node is self-evident.

\section{To establish the model of mine wireless communication}

Underground communication channel is different with the general communication channel, has its own characteristics[6].

Mine roadwayis a long and complex, some topological structure, simple star (star topology), any hop network type or grid type cannot simply structure suitable for mine. According to the actual situation, select the appropriate topology.

Coal mine tunnel as the channel of wireless data transmission is a kind of limited space longitudinal bend and branch. Communication is usually longitudinal transmission along the tunnel in tunnel.

This points out the particularity of coal mine underground channel, requirements for longitudinal transmission as far as possible in the limited space.

The coal mine production involves many aspects, need to have drainage, ventilation, power supply, supply pressure system, and the present situation of mine used at present is the coexistence of multiple system. How will this multi system unified into a set of integrated system, so as to realize the system, is a key problem in the application of wireless sensor networks.

To ensure adequate connectivity of network and additional network node, to solve the installation location of nodes in certain environmental conditions and installing number problem, namely the distribution of many nodes can meet the connectivity and coverage problem[7].

Because the system of coal mine production involves multiple, long front, such as mining equipment, fully mechanized equipment, belt transport, lifting machine, auxiliary transport system, etc.; also in the face of complex geological conditions, mine pressure, gas, carbon monoxide, underground water and coal dust, therefore, we will use the wireless sensor network in the mine for the three modes of the first application mode: can reach even often visited the occasions such as communication, lane, can replace a node one or two months[8]. Objective: to solve the communication problem of wireless relay in the confined space, the energy loss is not a research focus. The focus of the study is a self - organization, routing network, management etc..

Second kinds of application modes: people cannot or is not easy to reach the area of application, such as goaf, a variety of well bore, especially the air shaft and main shaft. In the limited space, how to solve the problem of energy efficiency is the main research content.

Third kinds of application modes: disaster relief communications applications, usually as a normal application of wireless sensor network, in the event of a major accident, into a disaster relief communication network. Research focus: the problem of transmission and network reconfiguration 
in a variety of different medium; the medium of variable case transmission and reconstruction problem.

Three kinds of application modes complement each other, wherein the first kind of application mode of the most basic and urgent, because the communication network roadway can be regarded as the backbone network in the underground wireless communication, while the second and the third kinds of application modes of network is formed by branch network, the special case of therefore, second and third mode in order to play a role, must be based on the work of the first kind of application mode on normal. As the wireless sensor network in the application study under just start, in this paper, we will focus on the research of mine wireless sensor network application mode in the first. 5 The introduction of Agent in Wireless Sensor Networks

Coal production has obvious particularity, before the safety the production process has the characteristics of local control, distributed decision making, provides a good space for the application of multi Agent technology in [9-10]. The following to control management as an example of a coal mine production, the simple application of the introduction of Agent in the environment of mine.

The composition of production and safety system of each coal mine is not completely consistent, safety production executive system subsystems should treat specific according to the specific situation. In order to control management as the executive level of coal mine production subsystem, such as the working face system, substation system, hoist system; coordination level Agent according to the system function, geographical position is provided with 4 coordinate Agent, such as the mine production safety production monitor Agent, Agent, Agent, ground power supply monitoring system A-gent; tissue Agent refers to the ground dispatching center corresponding production control Agent (i.e. related computer system). The sub system with hierarchical structure, composed of Agent unit of a union way, realize the related tasks.

\section{Conclusion}

In order to realize the application of wireless sensor network in coal mine environmental monitoring system, the paper analyzed the key problems of the application in the mine environment situation and characteristics, the characteristics of mine wireless communication in mine communication channel and wireless sensor network. Because in the mine production environment, security is a very important problem, therefore, good monitoring of mine environment, and the monitoring data processing and analysis is an important work. In this paper, the application of wireless sensor network in the mine environment monitoring system, and the establishment of the network structure and topology of mine environment suitable for the application of multi Agent, to monitor the wind speed, environment monitoring to dust, carbon monoxide, temperature, humidity and other parameters, achieves the purpose of safety monitoring.

\section{References}

[1]L. Sun,Z. Li and Y. Chen, Wireless sensor network [M].Beijing: Tsinghua university press ,2005:4-24.

[2]Q. Zhao ,X. Chen,New wireless communication system used in underground coal mine prospects[J].Coal mine electromechanical,2005(2):26-28.

[3]J. Zhu, Wireless sensor network technology. ZTE Communications [J] . 2004, 10( 10): 14 -15 . 
[4]S. Wang and J. Yan,Theory and application of wireless sensor network [M]. Beijing: Beihang University press, 2007.

[5]Y. Yu and O. Li, The theory, technology and implementation of wireless sensor network [M] . Beijing: National Defence Industry Press, 2008: 9.

[6]P. Sun,The characteristics of coal mine wireless transmission[J].Coal mine design,1999(4):20-22.

[7]T. He,Research on the access technology of infinite spreading coal mine integrated service network in the[D].Xuzhou:China University of Mining and Technology,2003.

[8]S. Zhang,Tent law and modeling of radio digital communication[J].Journal of China Institute of Communications,2002,23(11):41-50.

[9]K. Zhang and N. Sun,.Application of Agent technology in mine in management decision making[J].Computer and modernization,2002(10):29-31.

[10]Hairong Qi,Sitharama S Iyengar, Krishnendu Chakrabarty.Multiresolution Data Integration using Mobile Agent in Distributed Sensor Networks[J].IEEE Transactions on Systems,Man and Cybernetics Part C:Applications and Reviews,2001,31(3):383-390. 